\title{
Relación entre inversión y resultados deportivos de España y el Reino Unido en los últimos 20 años (1996-2016)
}

\section{Relationship between investment and sports results of Spain and the United Kingdom in the last 20 years (1996-2016)}

\author{
Josué González-Ruiz¹, Gema Torres-Luque², Antonio Roca-Cruz², Esther Puga González y y David Cabello-Manrique ${ }^{1}$
}

1 Universidad de Granada, 2 Universidad de Jaén, 3 EU La Inmaculada

\begin{abstract}
Resumen: El objeto del estudio es analizar cómo los ciclos económicos afectan a la inversión en el tercer sector del deporte y a los resultados deportivos internacionales. Se comparan los modelos de Espańa y Reino Unido. Se seleccionaron los resultados olímpicos de ambos países en los últimos 20 años (Sídney 2000; Atenas 2004; Pekín 2008; Londres 2012 y Río 2016). Los resultados muestran como España ha ido disminuyendo la inversión pública en deporte desde la crisis, pero ha logrado mantener sus resultados deportivos en los Juegos Olímpicos. Esto evidencia una posible mejora de los recursos públicos y de la generación de recursos propios. Reino Unido muestra una recuperación económica mucho más sólida, que ha potenciado sus resultados deportivos, casi doblando el número de medallas en el periodo poscrisis. Se constata una mayor eficacia y eficiencia del modelo de Reino Unido que necesita menos de la mitad de deportistas olímpicos para conseguir excelentes resultados, generando un coste de medalla por habitante un 50\% menor que Espańa.

Palabras clave: resultados deportivos, Juegos Olímpicos, federaciones deportivas, economía deporte, subvenciones.
\end{abstract}

Abstract: The purpose of this article is to analyze how economic cycles affect investment in Sport Federations and their international sport results. This study analyzes the models of Spain and the United Kingdom to establish a starting point for change and to identify a model to follow. A sample of the Olympic games during the last 20 years of both countries was taking (Sidney 2000; Athens 2004; Beijing 2008; London 2012 and Rio 2016). The results show how Spain has been decreasing public investment in sports since the crisis, although maintaining its sport results. This shows an improvement of public resources and its own resources. UK shows a much stronger economic recovery, which has boosted its sports results as it has almost double the medal number in the post crisis period. There is a greater effectiveness and efficiency of the UK model that needs to send less than half Olympic athletes to get excellent results, generating a medal cost per inhabitant $50 \%$ lower than Spain.

Key words: sport scores, Olympic games, sports federations, sport economy, sports subsidies.

\section{Introducción}

En los Juegos Olímpicos (JJOO) modernos, la guerra fría supuso que ambos bloques centraran sus políticas, en la obtención del mayor número de medallas olímpicas, con el objetivo de demostrar su superioridad no solo deportiva, sino también económica, política y logística (Rodríguez-Quijada, 2017; Chinchilla, 2009). Estos objetivos continúan latentes en la actualidad, ya que el éxito deportivo, se asocia a la proyección internacional del país, considerando, además, que los Juegos Olímpicos son uno de los acontecimientos deportivos y social es más destacados a nivel mundial (Brundage, 1973; Olivera, 2012), donde las gestas tienen un gran impacto en la vida de los seres humanos asentándose en su memoria (Bucur, Macovei, \& Margineantu, 2015).

En este sentido, en las sociedades occidentales, el deporte se estructura en torno a dos modelos bien diferenciados: el anglosajón, caracterizado porque en él prima la iniciativa privada de marcado tinte liberal en términos económicos y, por otra parte, el que se denomina europeo-continental, donde existe una fuerte intervención pública, ya sea de forma cen-

Dirección para correspondencia [Correspodence address]: David CabelloManrique. Universidad de Granada. E-mail: dcabello@ugr.es tralizada, como es el caso Francia, o descentralizada, el caso de España. Según Scheerder, Willem \& Claes (2017), desde 2009 como resultado de la crisis económica, especialmente dura en España, los presupuestos para deporte han descendido.

Así, el estudio comparado del sistema español con algunos de los países más importantes de la Unión Europea (UE) lleva a la necesidad de profundizar en este análisis para definir aquellas propuestas, iniciativas y modelos que podrían tener una traslación al sistema deportivo español con el objetivo de implementar aquellas medidas que están teniendo éxito en estos países, para así, mejorar la eficiencia del sistema, la coordinación y gestión del tejido asociativo, la organización y excelencia de las federaciones deportivas y, en definitiva, sobre su repercusión en el alto rendimiento y la representación internacional (Andreff, 2001)

Los objetivos fundamentales de ese estudio son analizar cómo los ciclos económicos afectan a los resultados deportivos de un país y la relación existente entre la inversión en el tercer sector del deporte y los resultados deportivos internacionales. Este estudio analiza los modelos español y del Reino Unido para establecer un punto de partida del cambio cuyo 
destino es la mejora, actualización y el establecimiento definitivo de un modelo identificativo basado en la contemporaneidad como sistema predominante.

\section{Material y método}

Se seleccionaron las olimpiadas de los últimos 20 años ( $n=5$ Juegos Olímpicos),en relación a España y Reino Unido. Los resultados deportivos se analizaron junto con el dinero público recibido en ese ciclo olímpico en cada país, para preparar la participación en los últimos 5 JJOO, Sídney 2000; Atenas 2004; Pekín 2008; Londres 2012 y Río 2016 (para Sidney 2000 se analizaron las subvenciones recibidas desde 1997 a 2000, los 4 años del ciclo, y así para cada caso). Los datos se recopilaron de entidades y organismos de carácter público y privado, mediante la consulta directa a entidades deportivas responsables, así como accediendo a sus documentos oficiales.

En la tabla 1 se muestran, las fuentes consultadas para cada país y sus enlaces web.

Tabla 1. Resumen de fuentes utilizadas para obtención de datos en Espańa y Reino Unido con sus enlaces web.

\begin{tabular}{|c|c|c|c|}
\hline \multicolumn{2}{|l|}{ España } & \multicolumn{2}{|l|}{ Reino Unido } \\
\hline Fuente & web & Fuente & web \\
\hline Consejo superior de deportes (CSD) & www.csd.gob.es & UK Sport (UKS) & www.uksport.gov.uk \\
\hline \multicolumn{2}{|c|}{ Instituto Nacional de Estadística (INE) www.ine.es } & Uk Government & www.gov.uk \\
\hline Federaciones Españolas (FF.EE) & $\begin{array}{l}\text { www.csd.gob.es/csd/ } \\
\text { asociaciones/1fedagclub/soap- } \\
\text { cli.2007-02-14.7388867167/ }\end{array}$ & England sports federation (NSGB) & www.uksport.gov.uk/ \\
\hline Comité Olímpico Espańol (COE) & www.coe.es & British Olympic Association (BOA) & www.teamgb.com/about-boa \\
\hline Web especializada Investing & www.investing.com & Web especializada medals per capita & www.medalspercapita.com/ \\
\hline
\end{tabular}

Se analizaron las siguientes variables:

a) Las medallas obtenidas por cada país en los JJOO de los últimos 20 años.

b) El Producto Interior Bruto (PIB) per cápita de cada país en cada ańo olímpico, expresada en miles de euros. Los datos económicos obtenidos de Reino Unido estaban cuantificados en Libras, por lo que fue necesario convertirlos a Euros, se tomó como referencia el valor de cambio establecido en el mes de junio de cada uno de los años de los que se obtuvieron los datos, para ello se consultó la página especializada https://es.investing. com.

c) La población total de cada país, expresado en millones de habitantes.

d) El coste público (Subvención)a las federaciones con disciplinas olímpicas en cada ciclo olímpico, medida en millones de euros.

e) El coste de cada medalla por ciclo olímpico, que se obtuvo dividiendo la subvención total de cada ciclo olímpico a las federaciones, entre el número de medallas obtenidas en dicha olimpiada y fue medida en millones de euros.

f) El coste medio de cada medalla por habitante, se obtuvo partiendo del coste público de cada medalla en su ciclo olímpico, se dividió este importe entre el número de habitantes del país y fue medida en euros.

g) El peso de cada medalla, para lo cual se utilizó el sistema propuesto por New York Times(https://beijing2008. blogs.nytimes.com/2008/08/23/the-medal-rankings- which-country-leads-the-olympics/) y aceptado por otras webs especializadas (http://www.medalspercapita.com/\#weighted-per-capita:2016) y organismos deportivos y que consiste en asignar un valor numérico al metal de la medalla obtenida, lo que proporciona una idea ponderable de la importancia de las medallas logradas por cada país; oro $=4$ puntos, plata $=2$ puntos, bronce $=1$ punto.

h) El número de deportistas olímpicos que necesita enviar cada país para conseguir una medalla. Esta variable, se determinó dividiendo el numero de deportistas enviados por cada país a cada JJOO entre el número de medallas conseguidas por cada país, obteniendo una ratio de deportistas por cada medalla obtenida.

i) La Influencia del periodo económico de cada JJOO, para lo cual, se dividió cada una de las Olimpiadas analizadas en relación al momento económico en donde se desarrollaron, de esta forma los JJOO de Sídney 2000, Atenas 2004 y Pekín 2008 fueron clasificadas como Pre-crisis (periodo 2000 a 2011) que incluye el periodo más duro de la crisis económica, y los JJOO de Londres 2012 y Río 2016, se clasificaron como Postcrisis (periodo 2012 a 2016).

Se empleó el paquete estadístico SPSS, versión 20.0 para Windows (SPSS, Armonk, New York). Se realizó un análisis descriptivo de cada una de las variables analizadas, donde se muestra media y desviación típica. Para observar las posibles diferencias entre los dos países seleccionados (España vs 
Reino Unido), y entre el momento de desarrollo de los JJOO (Pre-crisis vs Post-crisis) se empleó la prueba T Student para muestras independientes. Se consideró un nivel de significación al 95\%.

\section{Resultados}

A continuación se presenta la Figura 1, donde se aprecia la evolución de las medallas conseguidas desde Sídney 2000 a Rio 2016 y que se usa a modo introductorio, haciendo balance de los resultados obtenidos por ambos países.

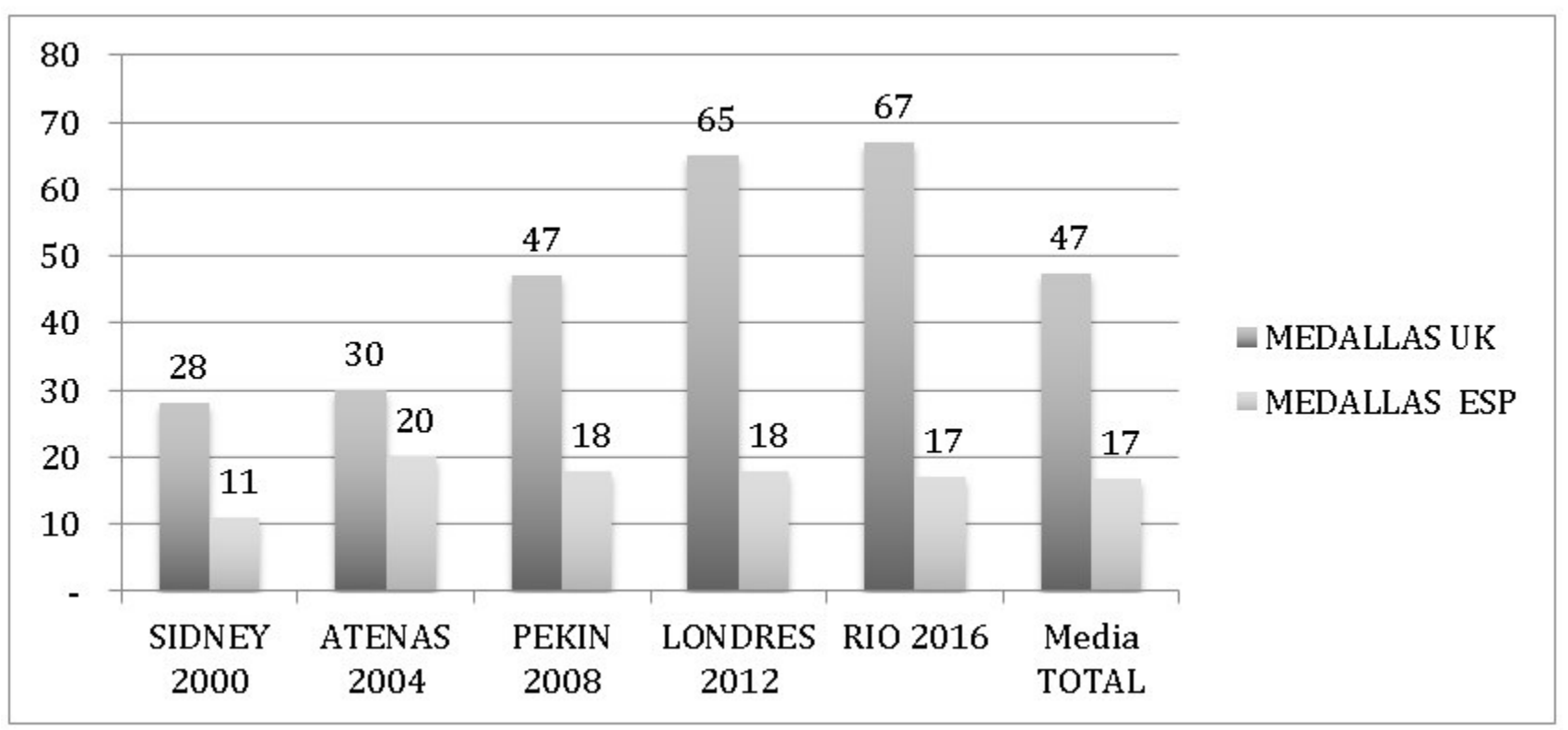

Figura 1. Evolución de las medallas conseguidas desde Sídney 2000 a Rio 2016.

La gráfica refleja como Espańa sólo ha conseguido mantener la media de medallas en los últimos JJOO apreciándose una ligera tendencia descendente, mientras que en el caso de Reino Unido el número de medallas ha crecido de forma continuada desde los JJOO de Sídney 2000. Se aprecia también una elevada diferencia entre el número medio de medallas conseguidas por Reino Unido y España en estos últimos cinco Juegos Olímpicos.

En la tabla 2 se pueden observar las diferencias entre las últimas cinco olimpiadas desde Sídney 2000 a Rio 2016, comparando España versus Reino Unido.

Se observa como existen diferencias estadísticamente significativas en todas las variables analizadas, exceptuando la variable subvención pública por ciclo y medalla. Destaca, sin embargo, el importe medio de las subvenciones públicas recibidas por las federaciones deportivas del Reino Unido, que obtiene valores más altos que los obtenidos por parte de las federaciones españolas. Se aprecian valores claramente superiores en la obtención de medallas por parte de Reino Unido frente a las conseguidas por España.

En la tabla 3 se analizan las diferencias por países de las distintas variables, atendiendo a si las olimpiadas se realizaron antes o después de la crisis económica.
Tabla 2. Diferencias, entre Espańa y Reino Unido en los últimos 5 JJOO (2000-2016).

\begin{tabular}{lccc}
\hline & España & $\begin{array}{c}\text { Reino } \\
\text { Unido }\end{array}$ & $p$ \\
\hline Medallas obtenidas & 16.8 & 47.4 & \multirow{2}{*}{ (No) } \\
PIB per cápita & \pm 3.4 & \pm 18.5 & $p=.007$ \\
(Euros) & 22212 & 32500 & \\
Población total & \pm 3601 & \pm 2201 & $p=.001$ \\
(Millones habitantes) & 44.4 & 61.8 & \\
Subvención Publica & \pm 2.7 & 2.6 & $p=.000$ \\
(Millones de Euros) & 143 & 267 & \\
Subvención pública total del ciclo & \pm 37 & \pm 89 & $p=.020$ \\
y medalla (Millones de Euros) & \pm 0.8 & \pm 1.3 & $p=.068$ \\
Coste medalla habitante & 0.201 & 0.093 & \\
(Euros) & \pm 0.08 & \pm 0.11 & $p=.020$ \\
Peso Medallas & 36.6 & 119 & \\
(Puntos ponderados) & \pm 8 & \pm 50 & $p=.007$ \\
Deportistas olímpicos necesarios & 19 & 8 & \\
para conseguir medalla (No) & \pm 5 & \pm 2 & $p=.004$ \\
\hline
\end{tabular}


Tabla 3. Diferencias en los JJOO celebrados pre-crisis (2000-2011) frente a post-crisis (2012-2016) En Espańa y Reino Unido.

\begin{tabular}{|c|c|c|c|c|c|c|}
\hline & \multicolumn{3}{|c|}{ España } & \multicolumn{3}{|c|}{ Reino Unido } \\
\hline & Pre & Post & $p$ & Pre & Post & $p$ \\
\hline Medallas obtenidas (No) & $\begin{array}{l}16.3 \\
\pm 4.7\end{array}$ & $\begin{array}{r}17.5 \\
0.7\end{array}$ & $p=.764$ & $\begin{array}{c}35 \\
\pm 10.4\end{array}$ & $\begin{array}{c}66 \\
\pm 1.4\end{array}$ & $p=.029$ \\
\hline PIB per cápita (Euros) & $\begin{array}{l}21500 \\
\pm 4849\end{array}$ & $\begin{array}{l}23281 \\
\pm 1016\end{array}$ & $p=.659$ & $\begin{array}{l}31333 \\
\pm 1078\end{array}$ & $\begin{array}{l}34250 \\
\pm 2616\end{array}$ & $p=.165$ \\
\hline Población total (Millones habitantes) & $\begin{array}{l}42.9 \\
\pm 2.7\end{array}$ & $\begin{array}{l}46.6 \\
\pm 0.2\end{array}$ & $p=.165$ & $\begin{array}{l}60.1 \\
\pm 1.5\end{array}$ & $\begin{array}{c}64.4 \\
\pm 1\end{array}$ & $p=.041$ \\
\hline Subvención publica (Millones de Euros) & $\begin{array}{l}128 \\
\pm 22\end{array}$ & $\begin{array}{r}165 \\
\pm 53\end{array}$ & $p=.340$ & $\begin{array}{c}214 \\
\pm 70.4\end{array}$ & $\begin{array}{c}346 \\
\pm 30.7\end{array}$ & $p=.096$ \\
\hline Subvención pública total del ciclo y medalla (Millones de Euros) & $\begin{array}{l}8.4 \\
\pm 1\end{array}$ & $\begin{array}{l}9.4 \\
0.7\end{array}$ & $p=.800$ & $\begin{array}{c}6.1 \\
\pm 1.1\end{array}$ & $\begin{array}{c}5.2 \\
\pm 0.7\end{array}$ & $p=.039$ \\
\hline Coste medalla Habitante (Euros) & $\begin{array}{c}0.20 \\
\pm 0.10\end{array}$ & $\begin{array}{c}0.20 \\
\pm 0.05\end{array}$ & $p=.994$ & $\begin{array}{c}0.10 \\
\pm 0\end{array}$ & $\begin{array}{c}0.08 \\
\pm 0\end{array}$ & $p=.006$ \\
\hline Peso Medallas (Puntos ponderados) & $\begin{array}{c}35 \\
\pm 10\end{array}$ & $\begin{array}{l}39 \\
\pm 4\end{array}$ & $p=.659$ & $\begin{array}{c}85 \\
\pm 27\end{array}$ & $\begin{array}{c}170 \\
\pm 1\end{array}$ & $p=.026$ \\
\hline Deportistas olímpicos para conseguir medalla ( $\mathrm{N}^{\circ}$ ) & $\begin{array}{l}20.3 \\
\pm 7.5\end{array}$ & $\begin{array}{c}17 \\
\pm 1.4\end{array}$ & $p=.596$ & $\begin{array}{c}9 \\
\pm 2\end{array}$ & $\begin{array}{c}6.5 \\
\pm 2.2\end{array}$ & $p=.272$ \\
\hline
\end{tabular}

Como se puede observar, no existe ninguna diferencia estadísticamente significativa en los JJOO pre-crisis y post-crisis en España. Sin embargo, en el caso de Reino Unido, existe un incremento significativo en las medallas obtenidas y el peso de las mismas $(p<.05)$; produciéndose un descenso en la subvención pública por medalla, el coste de la medalla por habitante y los deportistas necesarios para conseguir medalla $(p<.05)$.

En la tabla 4, observamos las diferencias entre España y Reino Unido en los JJOO celebrados en periodo pre-crisis y post-crisis.

Tabla 4. Diferencias entre Espańa y Reino Unido en los JJOO celebrados en periodo pre-crisis (2000-2011) y post-crisis (2012-2016).

\begin{tabular}{|c|c|c|c|c|c|c|}
\hline & \multicolumn{3}{|c|}{ Pre-crisis } & \multicolumn{3}{|c|}{ Post-crisis } \\
\hline & España & $\begin{array}{l}\text { Reino } \\
\text { Unido }\end{array}$ & $p$ & España & $\begin{array}{l}\text { Reino } \\
\text { Unido }\end{array}$ & $p$ \\
\hline Medallas obtenidas (No) & $\begin{array}{l}16.3 \\
\pm 4.7\end{array}$ & $\begin{array}{c}35 \\
\pm 10\end{array}$ & $p=.048$ & $\begin{array}{c}17 \\
\pm 0.7\end{array}$ & $\begin{array}{c}66 \\
\pm 1.4\end{array}$ & $p=.001$ \\
\hline PIB per cápita (Euros) & $\begin{array}{l}21500 \\
\pm 4849\end{array}$ & $\begin{array}{l}31333 \\
\pm 1078\end{array}$ & $p=.027$ & $\begin{array}{l}23281 \\
\pm 1016\end{array}$ & $\begin{array}{l}34250 \\
\pm 2616\end{array}$ & $p=.031$ \\
\hline Población total (Millones habitantes) & $\begin{array}{l}42.9 \\
\pm 2.7\end{array}$ & $\begin{array}{c}60.1 \\
1.5\end{array}$ & $p=.001$ & $\begin{array}{l}46.6 \\
\pm 0.2\end{array}$ & $\begin{array}{c}64.4 \\
\pm 1\end{array}$ & $p=.213$ \\
\hline Subvención pública (Millones de Euros) & $\begin{array}{l}128 \\
\pm 22\end{array}$ & $\begin{array}{l}214 \\
\pm 70\end{array}$ & $p=.113$ & $\begin{array}{l}165 \\
\pm 53\end{array}$ & $\begin{array}{l}346 \\
\pm 30\end{array}$ & $p=.053$ \\
\hline Subvención pública total del ciclo y medalla (Millones de Euros) & $\begin{array}{l}8.4 \\
\pm 1\end{array}$ & $\begin{array}{l}6.1 \\
\pm 1\end{array}$ & $p=.374$ & $\begin{array}{c}9.4 \\
\pm 0.7\end{array}$ & $\begin{array}{c}5.2 \\
\pm 0.7\end{array}$ & $p=.002$ \\
\hline Coste medalla habitante (Euros) & $\begin{array}{c}0.20 \\
\pm 0.10\end{array}$ & $\begin{array}{c}0.10 \\
\pm 0\end{array}$ & $p=.191$ & $\begin{array}{c}0.20 \\
\pm 0.05\end{array}$ & $\begin{array}{c}0.08 \\
\pm 0\end{array}$ & $p=.095$ \\
\hline Peso Medallas (Puntos ponderados) & $\begin{array}{c}35 \\
\pm 10\end{array}$ & $\begin{array}{c}85 \\
\pm 27\end{array}$ & $p=.044$ & $\begin{array}{l}39 \\
\pm 4\end{array}$ & $\begin{array}{c}170 \\
\pm 1\end{array}$ & $p=.001$ \\
\hline Deportistas olímpicos para conseguir medalla $\left(\mathrm{N}^{\circ}\right)$ & $\begin{array}{l}20.3 \\
\pm 7.5 \\
\end{array}$ & $\begin{array}{c}9 \\
\pm 2 \\
\end{array}$ & $p=.065$ & $\begin{array}{c}17 \\
\pm 1.4 \\
\end{array}$ & $\begin{array}{l}6.5 \\
\pm 2 \\
\end{array}$ & $p=.002$ \\
\hline
\end{tabular}

Al comparar ambos países en los JJOO del periodo pre-crisis, versus post-crisis, se observa como existen diferencias en un gran volumen de las variables analizadas tanto en un perio- do como en el otro, donde Reino Unido obtiene valores más altos, siendo más marcados en los JJOO post-crisis $(p<.05)$. 


\section{Discusión}

La importancia económica y el impacto social, que los resultados de los deportistas que compiten representando al equipo nacional tienen para cada país y su imagen internacional de marca, motiva a presentar el siguiente estudio en el que se realiza un análisis comparativo del modelo de gestión del tercer sector del deporte en Espańa, con el modelo con mejores resultados en Europa en los últimos tres JJOO, que es el de Reino Unido, 20 puesto en Rio 2016 y $3^{\circ}$ en Londres 2012 (https://www.olympic.org/olympic-games).Siguiendo a Sánchez, Baraja \&Alén(2013)“El deporte, a través de cualquiera de sus manifestaciones, constituye uno de los sectores con mayor importancia económica", la magnitud es tal que el impactico turístico generado por la celebración de grandes eventos deportivos ha llevado a la creación de una materia de investigación denominada turismo de eventos (Fernández, 2014). Por otra parte, la proliferación de eventos y promoción de instalaciones deportivas ha incentivado el uso de los instrumentos de análisis de impacto para predecir los flujos económicos esperados en las localidades de acogida. A su vez esto ha generado una utilización engañosa de estos análisis con el objetivo de inflar posibles resultados para obtener el apoyo económico de instituciones privadas y públicas (Barajas, Salgado\& Sánchez, 2012), por lo que se pone de manifiesto la necesidad de crear herramientas y organismos de medición y control eficientes que permitan dotar de transparencia y utilidad a los análisis y al seguimiento de las ayudas públicas. Sin embargo, no es menos cierto que la economía del deporte constituye un área relativamente nueva de investigación (Pedrosa\& Salvador, 2003), por lo que no son demasiados los estudios previos que analicen la inversión económica que se hace a nivel deportivo, con los resultados que se obtienen, aspecto primordial para generar estrategias futuras.

En este sentido, tras analizar los datos, se observa cómo, a pesar de que España cuenta con un mejor ratio de deportistas Olímpicos por habitante y que en el tamaño de los equipos olímpicos acorde con la población está equilibrado, el conjunto organizativo del deporte federativo olímpico en Reino unido, parece ser mucho más eficiente (Tabla 2). Reino Unido invierte más dinero en sus federaciones olímpicas y sus deportistas consiguen más medallas, pero el coste de la medalla por habitante británico es menos de la mitad del coste para un habitante espańol (Tabla 2). Esta eficiencia se pone de manifiesto al comparar el número de deportistas que cada país necesita enviar a los JJOO para conseguir una medalla, se requiere una media de 19 deportistas olímpicos españoles, mientras que en el caso británico, la cifra es de sólo ocho deportistas por medalla.

Además de la fortaleza económica de Reino Unido con respecto a España y su mayor facilidad a la hora de afrontar la crisis (Tabla 4), hay un claro punto de inflexión desde la entrada en vigor del nuevo sistema de financiación en Reino unido, por el cual, desde 1996 se transfiere un porcentaje de los beneficios obtenidos de la Lotería nacional (http://www. uksport.gov.uk/about-us/partners/the-national-lottery). Por lo tanto, podríamos decir que este aumento de financiación ha sido determinante en el fulgurante aumento en la obtención de medallas por parte de Reino (Figura 1), que se ha disparado de un rango medio de 20-30 medallas en JJOO anteriores a Sídney 2000, a las 60-67 de los dos últimos JJOO.

En el mismo periodo, Espańa ha mantenido su media habitual de unas 17 medallas, con un gran descalabro de resultados en los JJOO de Sídney 2000 donde obtuvo sólo 11 medallas, tras los buenos resultados JJOO de Atlanta (17 medallas) y de Barcelona 92 (mejor resultado histórico con 22 medallas). Nos preguntamos cual es el impacto de estas curvas tan diferenciadas tanto para la imagen exterior de ambos países, como la imagen proyectada a los habitantes del propio país y su influencia a la hora de incentivar el deporte base.

Esto es una reflexión importante, ya que se sabe que en los últimos 40 años, conseguir medallas olímpicas ha sido impulsado por cuatro factores: población, renta per cápita, el desempeño pasado (tradición deportiva) y un efecto de acogida por ser anfitrión de los JJOO (Williams, 2012). Si observamos la historia olímpica de España, sin duda el efecto acogida de los JJOO de Barcelona 92 fue el que impulso la consecución de medallas, pero este efecto de acogida también trajo de la mano importantes medidas, como la implantación de los planes ADO (Asociación, Deportes Olímpicos) del deporte español, que no ha vuelto a ser potenciada desde los JJOO de Barcelona 92 y cuyos resultados se estancaron. En el caso del deporte olímpico en Reino Unido, parece que el motivo principal de la excelencia en los resultados no es ninguno de los cuatro anteriores, ya que siempre ha gozado de una privilegiada posición económica como país, una población estable y ya había albergado JJOO con anterioridad.

Existen dos concepciones contrapuestas del concepto de deporte, mientras que en Estados Unidos el deporte queda reducido a la práctica deportiva organizada (competición, animación, etc.), en el ámbito europeo, su concepción es mucho más amplia, englobando a actividades físicas y deportivas que puedan ser realizadas de manera individual y no organizada. A su vez, y dadas las características de bien público que tiene el deporte por sus implicaciones sociales, sanitarias, educativas o culturales, los gobiernos han intervenido en una mayor o menor medida en su gestión y desarrollo.(Lera, 2010). Desde nuestro punto de vista esta concepción más amplia del deporte europeo, debe de ser fortalecida para asegurar la supervivencia de todos lo beneficios públicos. A su vez proponemos un refuerzo de este incentivo público mejorando más aún las ventajas fiscales para planes de mecenazgo privado como los ya existentes en España con la creación del plan ADO. 
$\mathrm{Al}$ analizar los resultados de España pre-crisis y post-crisis, (Tabla 3) no hay diferencia significativa en ninguna variable, lo que induce a pensar que en lo referente al deporte olímpico, España no ha salido de la crisis, es decir no hay un cambio significativo en el aumento de subvención pública que respalde la mejoría económica del país, como tampoco hay una mejora de los resultados.De hecho, las ayudas en España, al desarrollo del deportista de alto nivel de las federaciones olímpicas españolas durante el ciclo olímpico de Londres 2012, fue mayor que la efectividad de los deportistas, cuando participaron en las principales competiciones internacionales (De Carlos, Alén \& Pérez, 2017). Además, las diferencias en las distribuciones de eficiencia tienden a desaparecer en el último año del ciclo olímpico. Es decir, los deportistas olímpicos españoles rindieron mejor en eventos cercanos a la cita olímpica, aspecto que podría ser analizado en futuras investigaciones.

La configuración de una identidad local propia, supone un activo o aliciente para el desarrollo local y regional (Pasquinelli, 2013). Por ello entendemos que el deporte además de fomentar la salud, puede ser una palanca fundamental para generar valores positivos en los ciudadanos de un país, y potenciar mejoras de autopercepción, pertenencia e identidad como país y colectivo.

La generación y comunicación de identidades se masifican y se aceleran en nuestro siglo, gracias a los eventos mediáticos soportados en las nuevas plataformas tecnológicas de información y comunicación, teniendo como ejemplo de ello los JJOO de Beijin 2008. China es hoy un país moderno, que se postula a ser la primera potencia mundial a corto plazo. Para ello se utilizaron todos los recursos disponibles con la finalidad de ocultar la otra realidad del país, la desigualdad económica, la contaminación ambiental, la mínima transparencia en el manejo de la información o la violación sistemática de los derechos humanos (Lemus, 2010). Como vemos la asociación de valores positivos a la marca del país, es tan poderosa que ha sido históricamente utilizada, tanto para lavar la imagen en países con dudosa reputación internacional, como para asociar los valores positivos del deporte a la marca de un país. Sin duda la imagen de la marca Espańa, se ha visto afectada durante la crisis económica, invertir en deporte sería una muy buena oportunidad para volver a asociar la marca España a valores como esfuerzo, superación y éxito.En esta línea, no hay que olvidar que las federaciones deportivas tienen un fin social, cuya motivación principal no es el ánimo de lucro, si no generar cambios sociales y ser reconocidas en su labor de desarrollo y promoción del deporte que representan (Smith, 2000).

Sin embargo al comparar el mismos periodos pre y post crisis en Reino Unido (Tabla 3), las diferencias son altamente significativas $(p<.05)$ en casi todas las variables analizadas, por lo que podemos deducir que la salida de la crisis parece ser mucho más sólida, aumentando muy significativamente el número de medallas conseguidas en el periodo post crisis, llegando casi a duplicarse con respecto al periodo pre crisis.

Además de la fortaleza económica británica, otra explicación es que el modelo de gestión británico y su estructura deportiva sean más eficientes y eficaces que en el modelo español, por lo que convendría estudiar en más profundidad las diferencias normativas y estructurales de su modelo, observando campos como la cualificación de los profesionales que interactúan con el deporte base, la conexión del deporte escolar con el deporte rendimiento o las horas dedicadas a la educación física, tanto desde el currículo propuesto por el sistema educativo, como por otros entes promotores de actividad física, salud u ocio.

Por otra parte debemos analizar también la posible ineficiencia del modelo espańol, que previo a la crisis de 2008, contaba con niveles de financiación más cercanos a los de Reino Unido (Tabla 4), y cosechó resultados por debajo del equipo británico. Esta tendencia parece haber cambiando, ya que a pesar de la importante disminución de subvenciones durante estos años de crisis, las federaciones españolas, han conseguido mantener el número de medallas, lo que nos induce a pensar a que se han conseguido un mayor nivel de eficacia y eficiencia, tanto en el desarrollo de recursos económicos propios como en la gestión de su personal, instalaciones y deportistas.

Por último sería interesante ver si el que España haya podido mantener un número de medallas con una inversión menor desde la crisis se debe a la efectividad de programas destinados al incentivo de deporte femenino, cuyos resultados en los JJOO se ha incrementado de forma notable en los últimos años.

\section{Conclusiones}

- Se observa como Reino Unido invierte más dinero en sus federaciones deportivas, pero la ratio coste de una medalla por habitante, es mucho más eficiente que en España.

- En España, no hay diferencia significativa en ninguna variable en cuanto a los JJOO pre-crisis versus postcrisis; mientras que en Reino Unido si se muestran diferencias al alza entre un momento y otro.

- Las diferencias entre Reino Unido y España en los JJOO pre-crisis son patentes, acentuándose cuando se observan las diferencias en los JJOO post-crisis.

- El conjunto organizativo del deporte federativo olímpico en Reino unido, resulta más eficiente que el español, aunque serían necesarios más análisis para poder determinar las causas. 
En el modelo español, las competencias del deporte de competición en las disciplinas olímpicas son responsabilidad de las distintas federaciones deportivas. En consecuencia, para promover todos los valores y materializar el enorme potencial que el deporte puede aportar a nuestro país y a la sociedad, parece lógico dotar de más instrumentos y profesionales para su adecuado funcionamiento y el control eficiente de dichos recursos.

\section{Agradecimientos}

Esta investigación ha sido financiada por el Ministerio de Educación, Cultura y Deporte, a través de las ayudas Salvador de Madariaga, referencia no: PRX16/00367.

\section{Referencias Bibliográficas}

1. Andreff, W. (2001). Los intentos europeos y franceses de elaborar una contabilidad nacional de la economía del deporte, en Otero Moreno, J. M. (director), Incidencia económica del deporte, Instituto Andaluz del Deporte, Málaga, 23-53.

2. Barajas, A., Salgado, J. \& Sánchez, P. (2012). Problemática de los estudios de impacto económico de eventos deportivos. Estudios de Economía Aplicada, 30 (2), 441-462.

3. Bucur, M. M., Macovei, S. \& Margineantu, G. S. (2015). Sustainable development in the context of the olympic games. Ovidius University Annals, Series Physical Education \& Sport/Science, Movement \& Health. 15 (2), 111-116.

4. Brundage, A. (1973). Prefacio en Coubertein, P. Ideario Olímpico (P.I). Madrid: Instituto Nacional de Educación Física.

5. Chinchilla-Marín, C. (2009). Los juegos olímpicos: la elección de la sede y otras cuestiones jurídicas. CizurMenor (Navarra): Thomson Reuters-Civitas.

6. De Carlos, P., Alén, E. \& Pérez-González, A. (2017). Measuring the efficiency of the Spanish Olympic Sports Federations. European Sport Management Quarterly, 17(2), 210-225. doi: 10.1080/16184742.2016.1245769

7. Fernández, M.T. (2014). El impacto turístico de los eventos deportivos: un estudio de caso. Cuadernos de turismo, 33, 59-76.

8. Scheerder, J., Willem, A. \&Claes, E. (2017). Sport Policy Systems and Sport Federations, A Cross-National perspective. PalgraveMacmillan. DOI 10.1057/978-1-137-60222-0_1
9. Lera, F. (2010). Una aproximación al Deporte desde la Economía. El Papel de la Fundación Observatorio Económico del Deporte (FOED). Encuentro Nacional de Observatorios del Deporte, 3-5 Noviembre 2010, Sevilla.

10. Lemus, D. (2010). La construcción de la nueva imagen china a través de los medios: análisis de la ceremonia de inauguración de los Juegos Olímpicos de Beijing y el desfile del 60 aniversario de la República Popular China. Confines de relaciones internacionales y ciencia política, 6(12), 51-76

11. Pasquinelli, C. (2013). 'Branding as Urban Collective Strategy-making: The formation of Newcastle Gateshead's Organisational Identity'. Urban Studies, 51, 1-17.

12. Pedrosa, R. \& Salvador, J.A. (2003). El impacto del deporte en la economía: problemas de medición. Revista asturiana de economía, 23, 61-84.

13. Sánchez, P.; Barajas, A. \& Alén, E. (2013). Los eventos deportivos como herramienta de promoción turística. Revista de Análisis Turístico, 16, 59-69.

14. Smith, D.R. (2000). Books Review. Journal of the American Society for Information Science, 51, 585-688

15. Williams, E. (2012). Jollygoodshow: Who will winthe 2012 Olympic Games in London?. Tuck Schol of Business at Dartmounth. 